\title{
Incidence and predictors of cutaneous manifestations during the early course of systemic sclerosis: a 10-year longitudinal study from the EUSTAR database
}

\author{
Elina G Wirz, ${ }^{1,2}$ Veronika K Jaeger, ${ }^{1}$ Yannick Allanore, ${ }^{3}$ Gabriela Riemekasten, ${ }^{4,5}$ \\ Eric Hachulla, ${ }^{6}$ Oliver Distler, ${ }^{7}$ Paolo Airò, ${ }^{8}$ Patricia E Carreira, ${ }^{9}$ Mohammed Tikly, ${ }^{10}$ \\ Serena Vettori, ${ }^{11}$ Alexandra Balbir Gurman, ${ }^{12}$ Nemanja Damjanov, ${ }^{13}$ \\ Ulf Müller-Ladner, ${ }^{14}$ Jörg Distler, ${ }^{15}$ Mangtao Li, ${ }^{16}$ Peter Häusermann, ${ }^{2}$ \\ Ulrich A Walker, ${ }^{1}$ EUSTAR coauthors
}

\begin{abstract}
Handling editor Tore K Kvien
- Additional material is published online only. To view please visit the journal online (http://dx.doi.org/10.1136/ annrheumdis-2015-207271)
\end{abstract}

For numbered affiliations see end of article.

\section{Correspondence to} Dr Ulrich A Walker, Department of Rheumatology, University Hospital Basel, Petersgraben 4, 4031 Basel, Switzerland;

ulrich.walker@usb.ch

EGW, VKJ, PH and UAW contributed equally.

Received 8 January 2015 Revised 15 May 2015 Accepted 13 July 2015 Published Online First 31 July 2015

\section{ABSTRACT}

Objectives To longitudinally map the onset and identify risk factors for skin sclerosis and digital ulcers (DUs) in patients with systemic sclerosis (SSC) from an early time point after the onset of Raynaud's phenomenon (RP) in the European Scleroderma Trials and Research (EUSTAR) cohort.

Methods 695 patients with SSc with a baseline visit within 1 year after RP onset were followed in the prospective multinational EUSTAR database. During the 10-year observation period, cumulative probabilities of cutaneous lesions were assessed with the Kaplan-Meier method. Cox proportional hazards regression analysis was used to evaluate risk factors.

Results The median modified Rodnan skin score (mRSS) peaked 1 year after RP onset, and was 15 points. The 1 -year probability to develop an $\mathrm{mRSS} \geq 2$ in at least one area of the arms and legs was $69 \%$ and $25 \%$, respectively. Twenty-five per cent of patients developed diffuse cutaneous involvement in the first year after RP onset. This probability increased to $36 \%$ during the subsequent 2 years. Only $6 \%$ of patients developed diffuse cutaneous SSc thereafter. The probability to develop DUs increased to a maximum of $70 \%$ at the end of the 10-year observation. The main factors associated with diffuse cutaneous SSc were the presence of anti-RNA polymerase III autoantibodies, followed by antitopoisomerase autoantibodies and male sex. The main factor associated with incident DUs was the presence of antitopoisomerase autoantibodies.

Conclusion Early after RP onset, cutaneous manifestations exhibit rapid kinetics in SSc. This should be accounted for in clinical trials aiming to prevent skin worsening.

\section{INTRODUCTION}

Systemic sclerosis (SSc) is a multisystem autoimmune disorder, characterised by vasculopathy and excessive tissue fibrosis. ${ }^{12}$ Skin sclerosis is a hallmark feature of the disease and is most commonly measured with the modified Rodnan skin score (mRSS) by assessing skin thickness in 17 different body parts. $^{2-4}$ Dependent on the distribution of skin sclerosis, patients are categorised, at the maximum of extent, as having either limited or diffuse skin involvement. ${ }^{5}$ Discrimination between limited and diffuse cutaneous SSc is important, as diffuse cutaneous SSc is associated with higher morbidity and mortality independent of autoantibody status. ${ }^{6}$ Digital ulcers (DUs) are cutaneous lesions caused by obliterative vasculopathy. Both, skin sclerosis and DUs have proven to highly impact daily living and quality of life. ${ }^{78}$

Several cross-sectional studies suggest that the prevalence of both skin sclerosis and DUs depends on sex, age and autoantibody status. ${ }^{6}$ 9-14 However, only few studies have prospectively investigated the impact of risk factors for cutaneous SSc lesions. ${ }^{11} 15$ Some of these studies were small, and others did not investigate DU incidence, were not able to capture the onset of skin sclerosis early during the disease course or were not able to calculate incidences.

Given the paucity of pivotal data on the temporal evolution of skin manifestations during the early course of SSc, our goal was to analyse the incidence of skin sclerosis and DUs in patients who developed SSc within 1 year after the onset of Raynaud's phenomenon (RP).

By using real-life data from the large multicentre European Scleroderma Trials and Research (EUSTAR) cohort, ${ }^{6}$ we also assessed the skin sclerosis in different body areas, and determined factors associated with an unfavourable outcome in terms of acquisition of diffuse skin involvement and DUs.

\section{METHODS}

\section{Study population and design}

The architecture of the multinational, prospective EUSTAR database has been described elsewhere. ${ }^{6} 16$ In order to be able to document clinical data of patients, each participating centre obtained ethical approval by its local ethics committee; written informed consent was acquired from each patient registered. The demographics and disease characteristics of patients collected between the database inauguration in June 2004 and the date of censoring (18 February 2014) were exported, provided that the patients were older than 18 years at their 
first EUSTAR visit and fulfilled the 1980 American College of Rheumatology (ACR) criteria for SSc. ${ }^{17}$ In order to capture patients early in their disease course, that is, to simulate an inception cohort, the analysis was restricted to patients who had their first EUSTAR visit within 1 year after RP onset.

Several outcome measures were analysed as a function of time after RP onset: the evolution of the mRSS, the presence of skin sclerosis (defined as an $\mathrm{mRSS} \geq 2$ points at the body area of interest), the presence of diffuse cutaneous involvement (defined as an mRSS $\geq 2$ points in at least one of the six skin areas proximal to the elbows and knees, ie, upper arms, chest, abdomen, thighs) and the presence of DUs (defined as ulcers distal to or at the proximal interphalangeal joints, and not thought to be due to trauma). The effect of sex, age (dichotomised at the median age at RP onset) and autoantibody status on skin sclerosis and DU incidence was assessed in more detail.

\section{Statistical analysis}

Frequencies and percentages of categorical variables were compared using Pearson's $\chi^{2}$ tests. Means and SDs, and for nonnormally distributed variables, additionally medians and IQRs, were reported; two-group comparisons were performed using Student's t tests or Wilcoxon-Mann-Whitney tests.

Using Kaplan-Meier (KM) methods, we assessed the time to the first diagnosis of the manifestations. The date of the visit at which these were first observed was used as the end time. In case the manifestation was already present at the first visit, the date of this first visit was regarded the end time. If the manifestation was never observed, the date of the last follow-up visit was regarded the censor time. KM estimates were compared by log-rank tests and incidence rates, and their 95\% CIs were calculated. Cox proportional hazards regression analysis was used to assess the combined effect of the potential risk factors sex, age and autoantibody status on disease manifestations. All data were analysed using Stata V.13.1 (Stata, Texas, USA).

\section{RESULTS}

\section{Patient characteristics}

At the time of the data export, the EUSTAR database included 9891 patients fulfilling the inclusion criteria. Of these, 695 patients had their first EUSTAR visit within 1 year of RP onset. These patients had a median observation time of 2.1 years (IQR 0.7-4.6; mean 3.1 years, SD 3.0). The patients included in our analysis were approximately 9 years older, had a higher baseline mRSS and had more frequently antitopoisomerase-I (anti-TOPO) or anti-RNA polymerase-III (anti-RNAP-III) autoantibodies than the patients excluded (table 1). Furthermore, the percentage of men was significantly higher in the group analysed than in the excluded group. In contrast, DUs and anticentromere autoantibodies (ACA) were less frequent.

\section{Skin sclerosis by body area}

We first aimed to describe the onset of skin sclerosis at different body areas (figure 1). Most patients developed skin sclerosis within the first year after RP onset. The probability of having an mRSS $\geq 2$ in at least one area of the upper extremities within the first year was significantly higher than for the lower extremities $(68.7 \%$, 95\% CI $63.8 \%$ to $73.6 \%$ vs $25.0 \%$, $95 \%$ CI $20.6 \%$ to $30.2 \%)$.

As expected, the highest incidence rate of skin sclerosis in the first year was observed at the fingers (105.7/100 patient-years, $95 \%$ CI 92.9 to 120.1$)$. At the more proximal areas of the upper extremities, the incidence in the first year was lower (figure 1A). The incidence rate of puffy fingers in the first year
Table 1 Comparison of disease characteristics at the baseline visit between patients included in this analysis (visit within 1 year after onset of RP) and those excluded (no visit within 1 year after onset of RP)

\begin{tabular}{|c|c|c|c|}
\hline & Included & Excluded & p Values \\
\hline \multicolumn{4}{|l|}{ Patient characteristics at baseline visit } \\
\hline Number of patients & 695 & 9196 & \\
\hline $\begin{array}{l}\text { Age at onset of RP, years; } \\
\text { mean (SD) }\end{array}$ & $51.7(14.2)$ & $42.4(14.8)$ & $<0.001$ \\
\hline Male, $\%$ & 26.6 & 13.3 & $<0.001$ \\
\hline \multicolumn{4}{|l|}{ Laboratory parameters per patient } \\
\hline ANA, $\%$ & 96.1 & 96.4 & 0.67 \\
\hline $\mathrm{ACA}, \%$ & 16.7 & 34.7 & $<0.001$ \\
\hline Anti-TOPO, \% & 42.0 & 33.2 & $<0.001$ \\
\hline Anti-RNAP-III*, \% & 9.5 & 2.9 & $<0.001$ \\
\hline \multicolumn{4}{|l|}{ Disease characteristics per patient } \\
\hline $\begin{array}{l}\text { Age at onset of first non-RP, } \\
\text { years; mean (SD) }\end{array}$ & $50.9(14.4)$ & $46.3(14.1)$ & $<0.001$ \\
\hline Digital ulcers, \% & 28.4 & 34.2 & 0.002 \\
\hline Puffy fingers, \% & 52.7 & 37.3 & $<0.001$ \\
\hline mRSS; median (IQR) & $10.0(4.0-19.0)$ & $6(3.0-12.0)$ & $<0.001$ \\
\hline mRSS; mean (SD) & $12.7(10.5)$ & $8.7(7.7)$ & $<0.001$ \\
\hline Diffuse cutaneous involvement, $\%$ & 20.6 & 9.0 & $<0.001$ \\
\hline
\end{tabular}

${ }^{*}$ Data on anti-RNAP-III autoantibodies are only available for 317 (45.6\%) of the included and 4187 (45.3\%) of the excluded patients.

ACA, anticentromere autoantibodies; ANA, anti-nuclear autoantibodies; anti-RNAP-III, anti-RNA polymerase-III autoantibodies; anti-TOPO, antitopoisomerase-I autoantibodies; mRSS, modified Rodnan skin score; RP, Raynaud's phenomenon.

tended to be lower than that of finger skin sclerosis $(90.4 / 100$ patient-years, 95\% CI 78.7 to 103.9), although this difference was not statistically significant. Similarly, the more distal areas of the lower extremities were more often affected than the more proximal areas (figure $1 \mathrm{~B}$ ).

In the central body areas (figure $1 \mathrm{C}$ ), the rate of skin sclerosis at the face was similar to that of the forearms (42.1/100 patientyears, 95\% CI 34.4 to 51.5). The chest and abdomen had rates similar to the thighs $(17.7 / 100$ patient-years, 95\% CI 13.0 to 24.1 and $12.8 / 100$ patient-years, $95 \%$ CI 8.9 to 18.4 , respectively).

All patients who developed moderate-to-severe skin sclerosis (defined as an mRSS $\geq 2$ at any body area) did so within 6.5 years after RP onset. The probability to develop skin sclerosis within this period was $87.7 \%$ (95\% CI $80.5 \%$ to $92.1 \%)$ in the upper extremities and $41.6 \%$ (95\% CI $33.6 \%$ to $50.8 \%$ ) in the lower extremities, $23.4 \%$ (95\% CI $16.5 \%$ to $32.7 \%$ ) at the chest and $22.0 \%$ (95\% CI $14.8 \%$ to $32.1 \%$ ) at the abdomen.

When applying a less stringent definition of skin sclerosis, namely a cut-off of $\geq 1 \mathrm{mRSS}$ point at any body area, the probability of developing skin sclerosis was $94.7 \%$ (95\% CI $88.5 \%$ to $98.2 \%)$; as in the case of the more stringent definition for skin sclerosis, all patients developed this within 6.5 years after RP onset. Thus, about $5.0 \%$ of patients had SSc sine scleroderma.

We also analysed the time of mRSS peaking in patients with skin involvement who had multiple visits with a documented mRSS $(\mathrm{n}=381)$. The median peak mRSS was 15 points (IQR 724; mean mRSS 16.7, SD 10.5) and was reached as early as 1 year after RP onset (IQR 0.6-2.4; mean 1.9 years, SD 2.2; see figure $2 \mathrm{~A}$ and online supplementary figure S1A). Patients with limited cutaneous involvement reached the mRSS peak of 9.5 points (IQR 6-14; mean mRSS 10.4, SD 6.1) after a median of 0.9 years (IQR 0.6-2.2; mean 2.0, SD 2.5; see figure $2 \mathrm{~B}$ and 


\section{Clinical and epidemiological research}
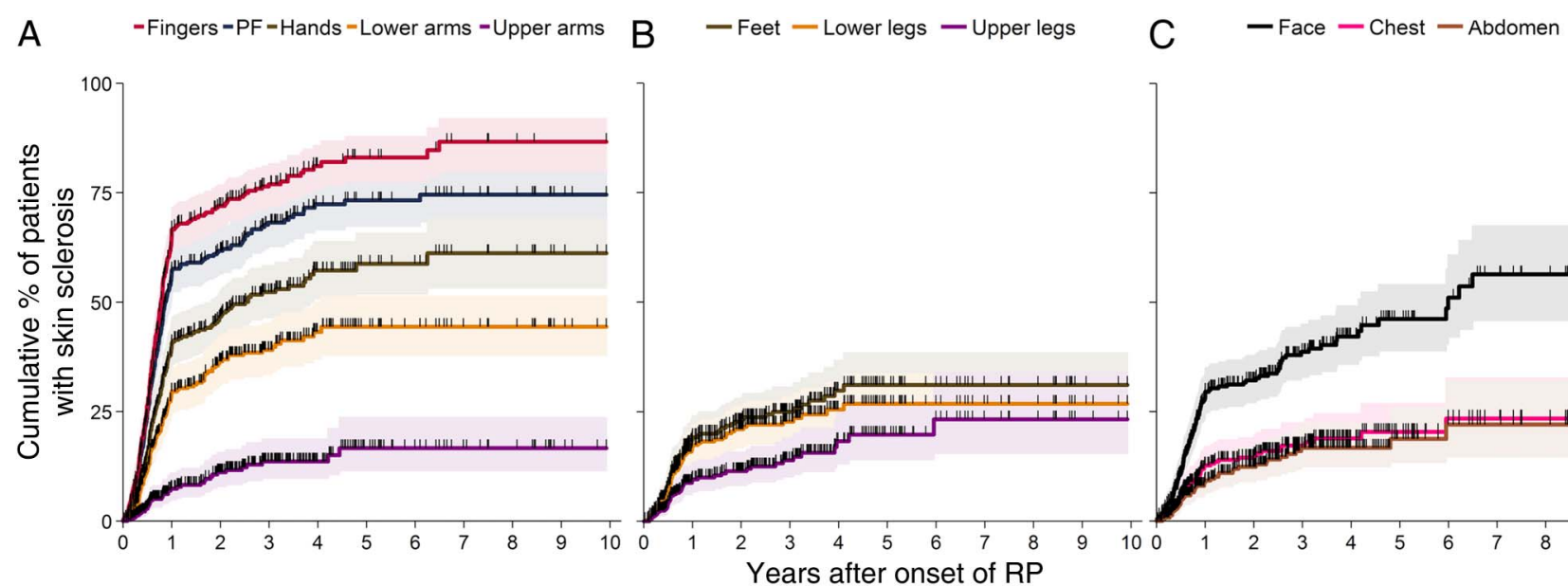

Figure 1 Kaplan-Meier curves with $95 \% \mathrm{Cl}$ of patients developing skin sclerosis defined as $\geq 2$ points at the area of mRSS scoring, separated for the upper extremities (A), the lower extremities (B) and the central body areas (C). mRSS, modified Rodnan skin score; PF, puffy fingers; RP, Raynaud's phenomenon; hash marks illustrate censored observations.

Figure 2 Time to peak mRSS. The histogram plots the percentage of patients as a function of the time to reach their maximal $m R S S$ from RP onset; for all patients (A) and divided into patients with limited cutaneous involvement and diffuse cutaneous involvement (B). The median peak mRSS was 15 points (IQR 7-24) overall, 9.5 points (IQR 6-14) in patients with limited cutaneous involvement and 23 points (IQR 1629.5) in patients with diffuse cutaneous involvement. mRSS, modified Rodnan skin score; RP, Raynaud's phenomenon.
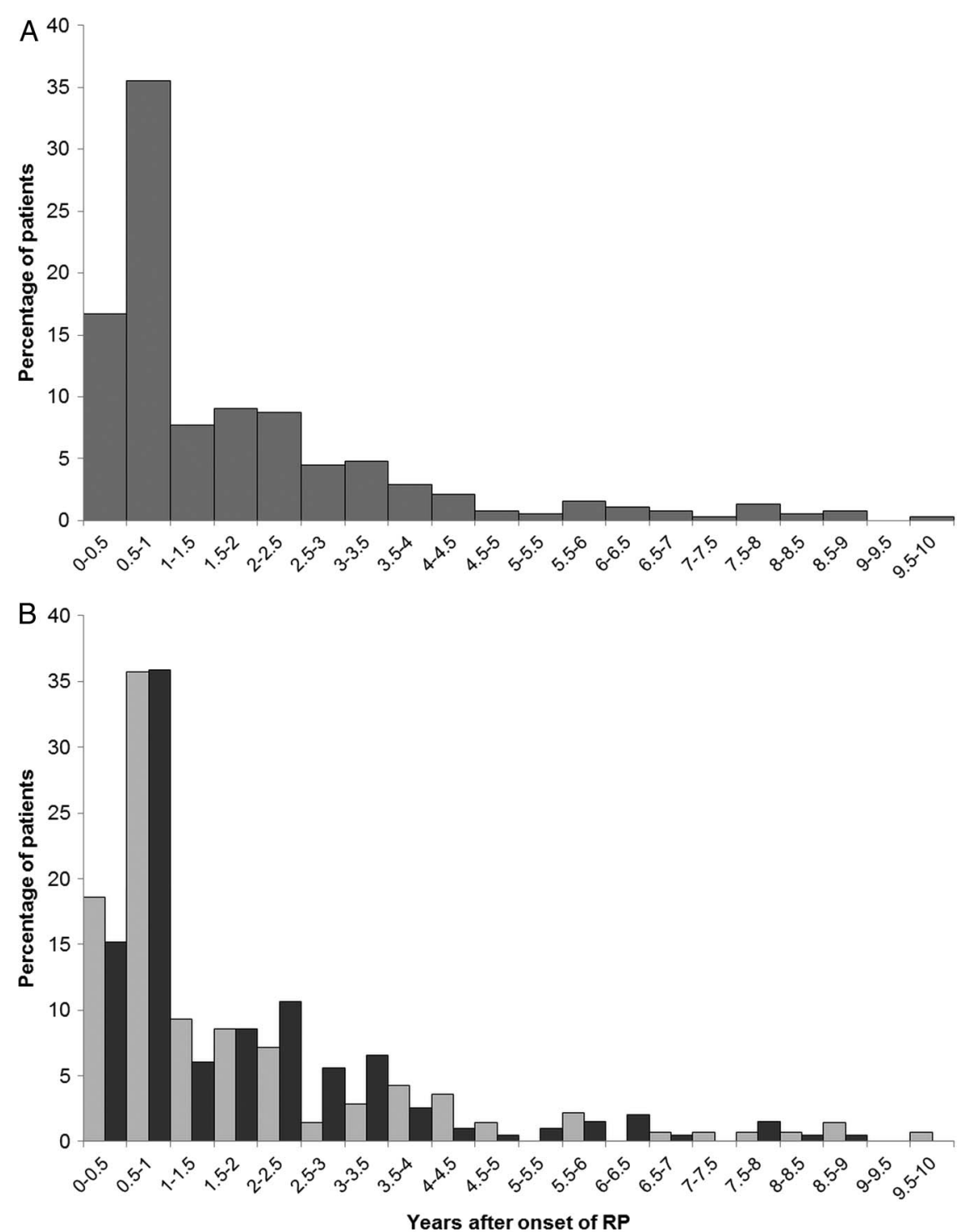
online supplementary figure S1B). For patients with diffuse cutaneous involvement, the peak mRSS was 23 points (IQR 1629.5 ; mean mRSS 22.8, SD 9.6) and was reached after a median of 1 year (IQR 0.6-2.5; mean 1.9 years, SD 2.0; see figure 2B and online supplementary figure S1B). Thus, there was no difference in the median time to reach the mRSS peak between patients with diffuse and patients with limited cutaneous SSc $(p=0.36)$. We also assessed other potential risk factors for the time to mRSS peaking in a multivariable analysis only including the first year after RP onset as half of the patients reached their maximal mRSS as fast as within this first year. In this analysis, neither patients' age and sex nor the presence of autoantibodies in the patients' sera was associated with the time to development of the maximal mRSS within the first year after RP onset (see online supplementary table S1).

\section{Skin sclerosis by mRSS severity}

Figure $3 \mathrm{~A}$ shows the probabilities to develop a total $\mathrm{mRSS}>5$, $10,20,30$ or 40 points. Only $1.2 \%$ of patients developed a total mRSS $>40$ points in the first year. In contrast, the probability of having $\leq 5 \mathrm{mRSS}$ points was $24.1 \%$ (95\% CI $20.7 \%$ to $27.6 \%$ ) in the first year.

Men had an almost twofold higher rate than women to develop an mRSS $>20$ points (rate ratio $1.8,95 \%$ CI 1.3 to 2.4 ) in the first year (figure 3B). Moreover, age at disease onset was found to be a predictor for severe skin involvement (mRSS $>20$ points) early during the disease course. The probability to develop an mRSS $>20$ points within 1 year was higher in older patients than in younger subjects when dichotomised at the median age (52.7 years, figure 3C). However, within the following 5 years, the probabilities of both age groups to develop severe skin involvement converged to approximately $42 \%$.

The analysis of the development of skin sclerosis by the presence of serum autoantibodies in the patients' sera revealed that all patients harbouring anti-RNAP-III autoantibodies and developing a total mRSS $>20$ points did so within 3 years after onset of RP (figure 3D); the 3-year probability was $76.4 \%$ (95\% CI $57.7 \%$ to 91.1\%). Patients with anti-TOPO autoantibodies also had a significantly higher probability of acquiring an mRSS $>20$ points in the first 3 years than patients with ACA $(44.8 \%$, 95\% CI $38.2 \%$ to $52.0 \%$ vs $2.5 \%, 95 \%$ CI $0.6 \%$ to $9.7 \%$, respectively).

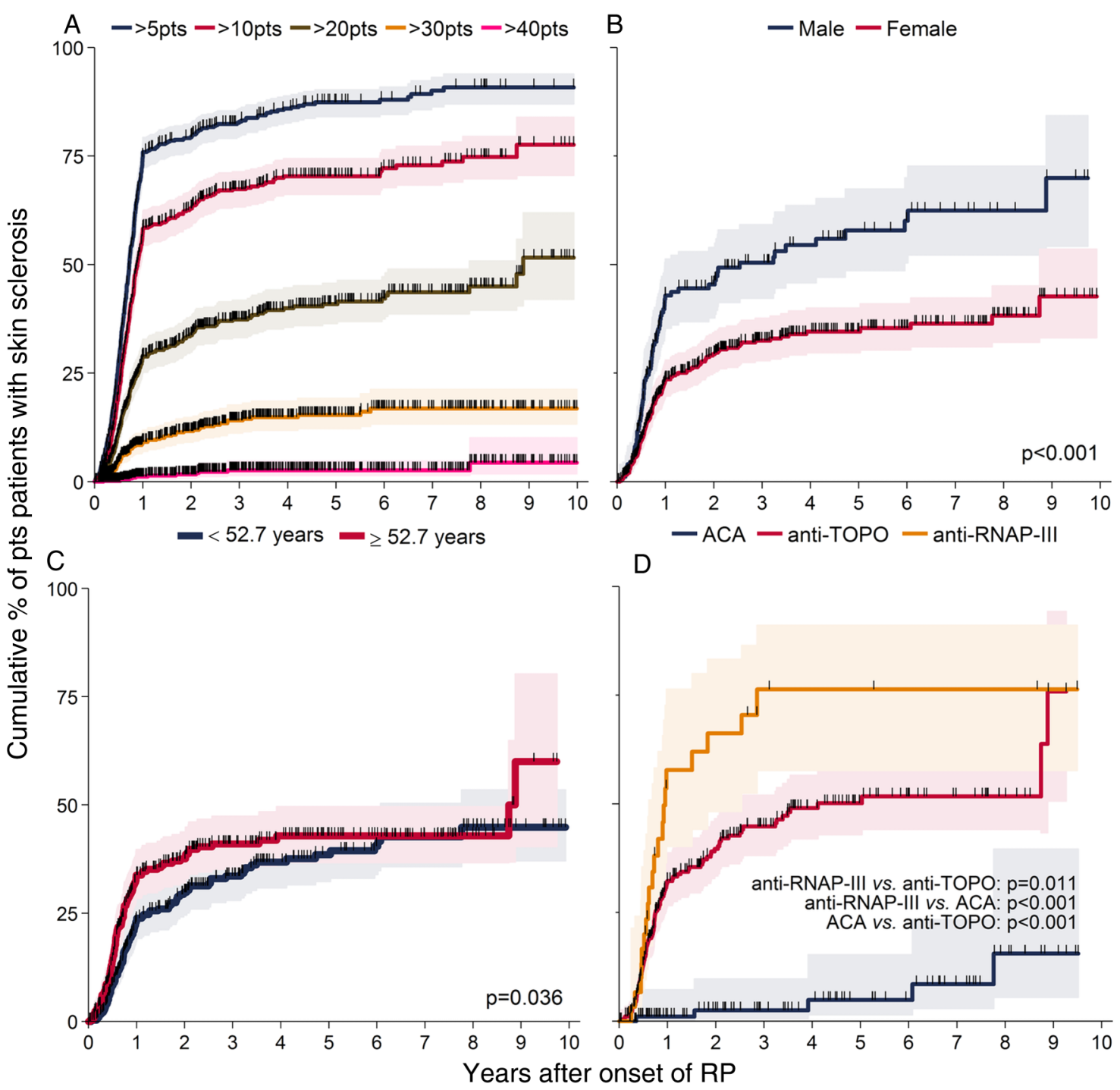

Figure 3 Kaplan-Meier curves with $95 \% \mathrm{Cl}$ of patients developing an mRSS $>5$ points, $>10$ points, $>20$ points, $>30$ points and $>40$ points after the onset of RP (A). Developing a total mRSS $>20$ points was further stratified by sex (B), by the median age at the onset of RP (C) and by their autoantibody status (D). ACA, anticentromere autoantibodies; anti-RNAP-III, anti-RNA polymerase-III autoantibodies; anti-TOPO, antitopoisomerase-I autoantibodies; mRSS, modified Rodnan skin score; pts, points; RP, Raynaud's phenomenon; hash marks illustrate censored observations. 


\section{Diffuse cutaneous involvement}

The probability to develop diffuse cutaneous involvement, defined as an mRSS $\geq 2$ at any area of the proximal extremities or trunk within the first year was $24.8 \%$ (95\% CI $20.2 \%$ to $30.3 \%$ ) increasing to $35.7 \%$ (95\% CI $29.9 \%$ to $42.3 \%$ ) during the subsequent 2 years (figure 4A). Only a minority of patients developed a diffuse cutaneous involvement beyond 3 years after RP onset.

The rate of developing diffuse skin involvement in the first year in men was more than twice than that in women (rate ratio 2.4, 95\% CI 1.5 to 3.96; figure 4B). There was, however, no evidence for differences in the probability of developing diffuse skin involvement between younger and older age groups (figure 4C).

With respect to autoantibody status, the rates of developing diffuse cutaneous involvement in the first 3 years were highest in patients with anti-RNAP-III autoantibodies (70.6/100 patientyears, 95\% CI 42.7 to 93.2 ), followed by those with anti-TOPO autoantibodies (43.2/100 patient-years, 95\% CI 33.1 to 54.9 ) and lowest in patients with ACA (2.3/100 patient-years, 95\% CI 0.3 to 15.1 ; figure $4 \mathrm{D})$.
In the multivariable analysis of potential risk factors for the development of diffuse cutaneous involvement, defined as signs and symptoms present within the first year after RP onset, the presence of anti-RNAP-III and anti-TOPO autoantibodies conferred an elevated risk (HR 16.9, 95\% CI 3.7 to 77.4 and 10.7, 95\% CI 2.5 to 44.9 ) compared with ACA. Furthermore, male sex was also confirmed as a risk factor for diffuse cutaneous involvement (HR 2.7, 95\% CI 1.6 to 4.7, see online supplementary data). However, neither patient's age at RP onset nor the presence of DUs or puffy fingers were associated with the development of diffuse cutaneous involvement.

\section{Digital ulcers}

There was a steep increase of DU development in the first year (figure 5A), with a probability of $33.7 \%$ (95\% CI $30.0 \%$ to $37.8 \%)$. Unlike skin sclerosis, the probability of patients to develop DUs increased continuously over the 10-year observational period to a maximum of $70.2 \%$ (95\% CI $60.4 \%$ to $79.5 \%$ ) after the initial steep increase in the first year. The median time to DU development among those patients who

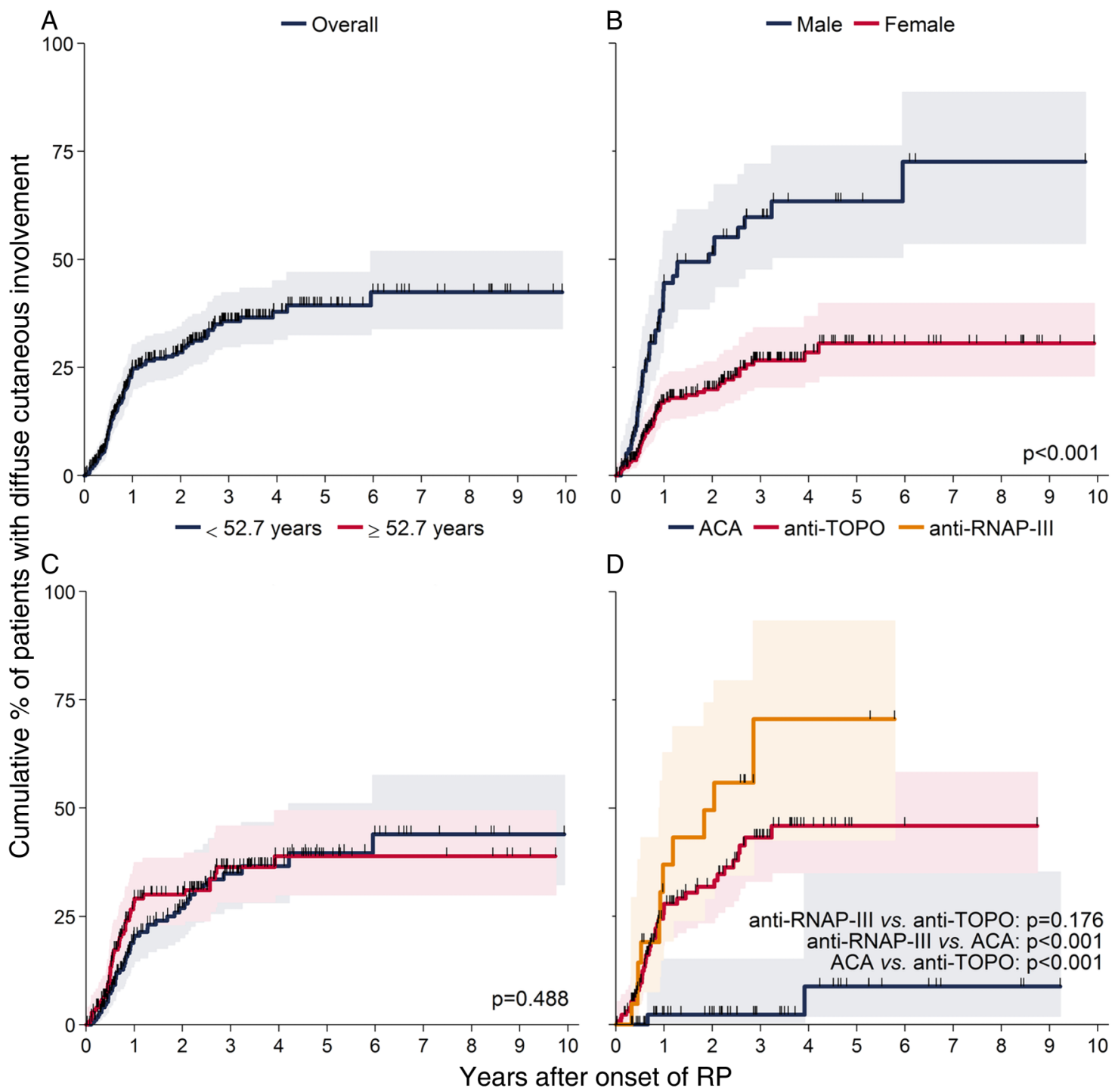

Figure 4 Kaplan-Meier curves with $95 \% \mathrm{Cl}$ of the first reported diffuse cutaneous involvement after the onset of RP in all patients in this analysis (A), stratified by sex (B), by the median age at the onset of RP (C) and by their autoantibody status (D). ACA, anticentromere autoantibodies; anti-RNAP-III, anti-RNA polymerase-III autoantibodies; anti-TOPO, antitopoisomerase-I autoantibodies; RP, Raynaud's phenomenon; hash marks illustrate censored observations. 


\section{Clinical and epidemiological research}

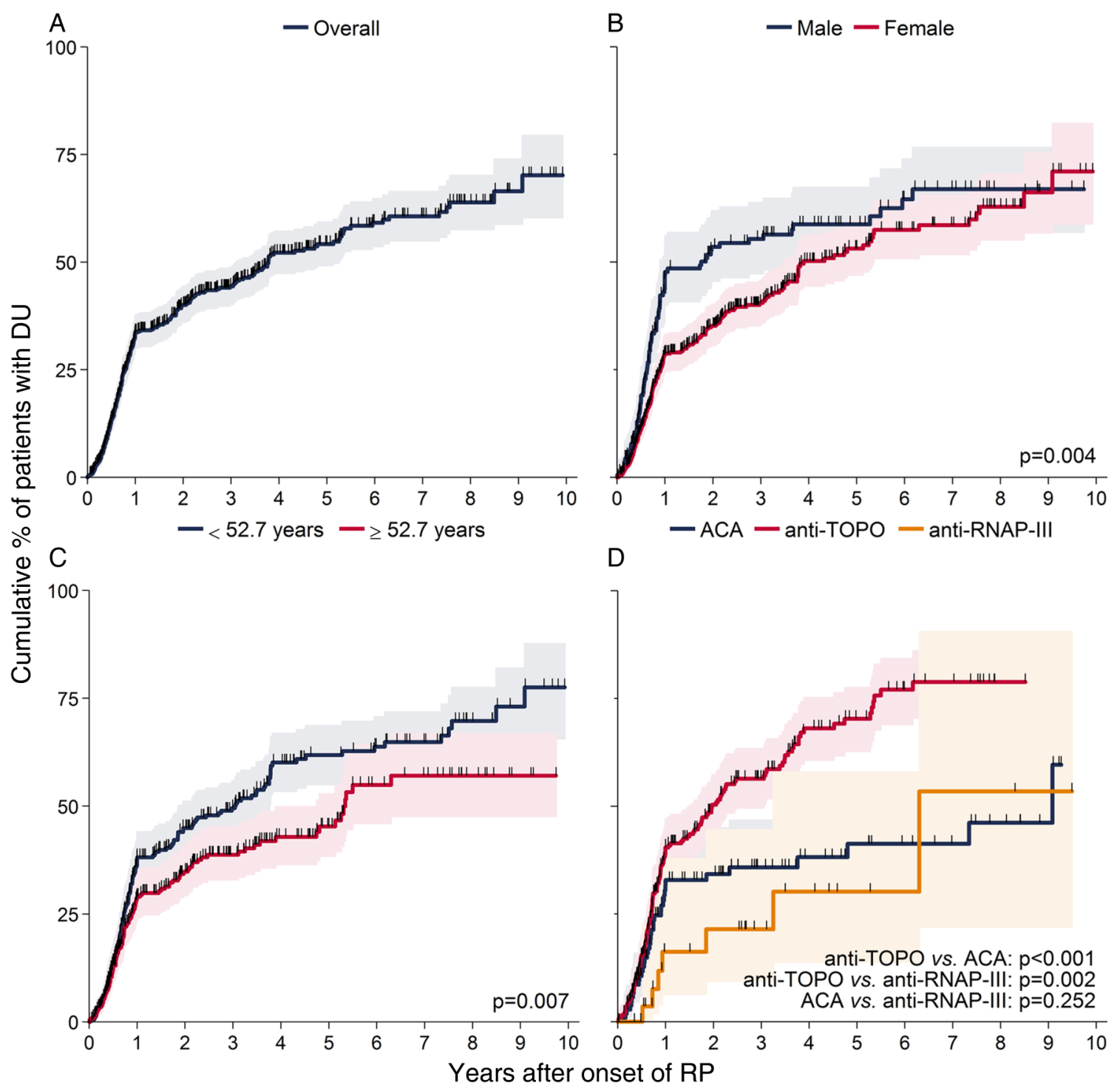

Figure 5 Kaplan-Meier curves with $95 \% \mathrm{Cl}$ of the observed first DU after the onset of RP (A), stratified by sex (B), by the median age at the onset of RP (C) and by their autoantibody status (D). ACA, anticentromere autoantibodies; anti-RNAP-III, anti-RNA polymerase-III autoantibodies; anti-TOPO, antitopoisomerase-I autoantibodies; DU, digital ulcers; RP, Raynaud's phenomenon; hash marks illustrate censored observations.

acquired DUs was short (0.7 years, IQR $0.4-1.7$; mean 1.4 years, SD 1.6).

Men had a significantly higher incidence rate of DUs within the first year than women (66.5/100 patient-years, 95\% CI 53.0 to 83.6 vs 42.0 patient-years, $95 \%$ CI 35.2 to 50.1$)$. After 6 years, however, the probabilities to develop DUs converged to approximately $60 \%$ in both sexes (figure $5 \mathrm{~B}$ ).

Younger patients tended to be affected by DU earlier and more frequently than older subjects (figure 5C). In contrast to skin sclerosis, patients with anti-RNAP-III had a lower probability to develop DUs than those with anti-TOPO autoantibodies (figure 5D). Of note, there was no difference in the probability between patients with anti-RNAP-III autoantibodies and those with ACA.

Only the presence of anti-TOPO autoantibodies was associated with DU development (HR 1.8, 95\% CI 1.2 to 2.6) in multivariable analysis, but not the presence of the other autoantibodies, age at RP onset, sex or the presence of puffy fingers (see online supplementary table S1).

\section{DISCUSSION}

This study has analysed prospectively the incidence of SSc skin manifestations in patients who present as early as 1 year after the onset of RP. An important finding is that most patients acquire the maximal gain of skin sclerosis within 1 year after RP onset and that diffuse cutaneous involvement emerges newly in only a minority of patients after 5 years of disease onset. This study, in which we also map the evolution of skin sclerosis at different body areas, underlines that the fibrotic process is most fulminant initially in all body areas, and is less active at later stages. The study by Steen and Medsger also highlighted that an mRSS $>40$ points mainly occurred in the first 3 years in patients with diffuse cutaneous SSc, but the study was not able to detail the evolution of skin sclerosis during the first 3-year period and to investigate the disease evolution in patients with an mRSS $<40$ points. $^{18}$

Our study confirmed that the presence of some autoantibodies is associated with the severity of skin sclerosis, as demonstrated in several cross-sectional studies. ${ }^{6} 1920$ Although two 
small cross-sectional studies suggested an association between anti-RNAP-III autoantibodies and diffuse cutaneous involvement or a higher mRSS, ${ }^{19}{ }^{20}$ none of these studies compared several autoantibodies simultaneously, and none of these studies highlighted the effect of anti-RNAP-III positivity on the incidence and mRSS kinetics during the early disease course.

It is still a matter of debate as to whether patients with late-age onset SSc are more likely to have limited or diffuse cutaneous SSc than patients with early-age onset SSc. ${ }^{12-14} 21$ Some investigators found no differences in the severity of skin involvement between age-of-onset groups. ${ }^{14}{ }^{22}$ In line with a previously published cross-sectional analysis of our cohort, younger age at SSc onset was not associated with a higher mRSS in this longitudinal analysis. ${ }^{6}$

The short median time to mRSS peaking and the fact that the mRSS gain is highest in the first year of disease, regardless of the magnitude of the maximal mRSS, suggest that SSc kinetics peaks within the first year in the skin despite inter-individual differences in the final severity of skin sclerosis. Interestingly, the risk factors governing the extent and severity of skin involvement (eg, male sex and autoantibody status being associated with the development of diffuse disease) were not found to influence the disease kinetics (eg, the time to mRSS peaking).

In contrast to the rapid initial evolution of skin sclerosis, the probability to develop DUs increased more continuously over time. In our study, only the presence of anti-TOPO, and not anti-RNAP-III autoantibodies, was associated with an increased incidence of DUs, compared with the presence of ACA. These findings are in line with several previous studies that suggest a higher prevalence of DU in patients with anti-TOPO autoantibodies compared with patients without these autoantibodies. ${ }^{6} 23$ A small retrospective cohort also suggested that patients with anti-RNAP-III autoantibodies had less peripheral vascular disease than patients with anti-TOPO autoantibodies, and similar rates to patients with ACA. ${ }^{24}$

Most studies agree in the observation that DUs are less prevalent in late-age than young-age onset patients. ${ }^{6} 9{ }^{10}{ }^{12-14}$ The univariate analysis of our prospective study also highlights a higher DU incidence in patients who are young at disease onset, but interestingly this association was lost after controlling for sex and autoantibody status.

The strengths of our investigation are the large sample size and the longitudinal multinational nature of our cohort. The mRSS score has been validated as a reliable outcome measure and demonstrated large effect sizes and sensitivity to change. ${ }^{3}{ }^{4} \mathrm{~A}$ somewhat smaller effect size must be taken into account when interpreting skin scores of isolated body sites, and the mRSS interobserver variability must be accounted for when interpreting cohort data. We also only analysed patients who had been recruited into EUSTAR within the first year after RP onset, thereby simulating an inception cohort. This selection and the fact that we only recruited patients who fulfilled the ACR classification criteria for SSc is, however, also a limitation of this study, as evidenced by the high prevalence of risk factors generally attributed to an adverse outcome (male sex and anti-TOPO positivity) in the patients included, as compared with those excluded. ${ }^{6} 1525$ As a consequence, the patients included had a comparatively higher median mRSS and more often diffuse cutaneous involvement at the baseline visit. Therefore, the results of our study must not be generalised to patients who present with SSc later than 1 year after RP onset. It should be also noted that the temporal evolution of skin manifestations might be underestimated, as some patients already had skin sclerosis at baseline. By including in this study only patients with a baseline visit within
1 year after RP onset, we tried to keep this effect low. In future studies, it may be interesting to follow patients who only meet the ACR/European League Against Rheumatism, and not the ACR classification, criteria for SSc to study drug effects.

Our study, nevertheless, critically contributes to the management of those patients with SSc who present early after RP onset. By mapping the temporal evolution of skin sclerosis and DUs and identifying risk factors early during the disease course, our findings will enable physicians to more accurately counsel patients with SSc presenting early. The long-term prospective data on the large number of EUSTAR patients presented here will facilitate the design of clinical trials aiming to prevent disease evolution as well as those evaluating new diagnostic tests and therapeutic strategies.
Author affiliations
${ }^{1}$ Department of Rheumatology, University Hospital Basel, Basel, Switzerland
${ }^{2}$ Department of Dermatology, University Hospital Basel, Basel, Switzerland
${ }^{3}$ Department of Rheumatology A, Paris Descartes University, Cochin Hospital, Paris, France
${ }^{4}$ Department of Rheumatology, Charité University Hospital, Berlin, Germany
${ }^{5}$ German Rheumatism Research Centre (DRFZ), Leibniz Institute, Berlin, Germany
${ }^{6}$ Department of Internal Medicine, Hôpital Claude Huriez, University Lille, Lille Cedex, France
${ }^{7}$ Department of Rheumatology, University Hospital Zurich, Zurich, Switzerland ${ }^{8}$ Rheumatology and Clinical Immunology Service, Spedali Civili di Brescia, Brescia, Italy
${ }^{9}$ Servicio de Reumatologia, Hospital Universitario 12 de Octubre, Madrid, Spain
${ }^{10}$ Division of Rheumatology, Chris Hani Baragwanath Academic Hospital, Faculty of
Health Sciences, University of the Witwatersrand, Johannesburg, South Africa
${ }^{11}$ Rheumatology Department, Second University of Naples, Naples, Italy
${ }^{12}$ B. Shine Rheumatology Unit, Rappaport Faculty of Medicine, Rambam Health Care Campus, Technion-Institute of Technology, Haifa, Israel
${ }^{13}$ Institute of Rheumatology, University of Belgrade Medical School, Belgrade, Serbia
${ }^{14}$ Department of Rheumatology and Clinical Immunology, Justus-Liebig University
Giessen, Kerckhoff Clinic, Bad Nauheim, Germany
${ }^{15}$ Department of Internal Medicine 3, University of Erlangen-Nuremberg, Erlangen,
Germany
${ }^{16}$ Department of Rheumatology, Peking Union Medical College Hospital, Chinese Academy of Medical Sciences, Beijing, China

Acknowledgements EUSTAR acknowledges the unconditional support that EULAR has provided in the past for the maintenance of the EUSTAR database.

Collaborators EUSTAR coauthors: Lidia Ananieva, Stefan Heitmann, Simona Rednic, Sergio Jimenez, Valeria Riccieri, Magdalena Szmyrka-Kaczmarek, Dominique Farge, Giovanni Lapadula, Marco Matucci-Cerinic, Serena Guiducci, Nicolas Hunzelmann, Maria Rosa Pozzi, Carina Mihai, Douglas Veale, Roger Hesselstrand, Eduardo Mariok, Vanessa Smith, Eugene J Kucharz, László Czirják, Duska Martinovic, Kamal Solanki, Codrina Mihaela Ancuta, Jean Sibilia, Caramaschi Paola, Manal Hassanien, Sarah Kahl, Adrianne Woods, Marie Vanthuyne, Ionescu Ruxandra, Sebastião C Radominski, Andrea Lo Monaco, Ada Corrado, Michaela Koehm, Montecucco Maurizio, Bevcar Radim, Esthela Loyo, Maria Üprus, Raffaele Pellerito, Thierry Zenone, Armando Gabrielli, Otylia Kowal-Bielecka, Blaz Rozman, Raffaella Scorza, Lesley Ann Saketkoo, Oyvind Midtvedt, Carlos Alberto von Mühlen, Jörg Henes, Ani Branimir, Paul Hasler, Sule Yavuz, Peter Villiger, Brigitte Krummel-Lorenz, Magdalena Posa, Merete Engelhart, Christopher Denton, Dorota Krasowska, Paloma Garcia de la Peña Lefebvre, Franco Cozzi, Luc Mouthon, Sergio Jimenez, Edoardo Rosato, Selmi Carlo, Juan José Alegre Sancho, Carmel Mallia, Massimiliano Limonta, Matthias Seidel, Rosario Foti, Lisa Stamp, Susanne Ullman, Simon Stebbings, Vera Ortiz Santamaria, Francesco Del Galdo, Ellen De Langhe, Alessandro Mathieu, Cord Sunderkötter, Kilian Eyerich, Bojana Stamenkovic, Srdan Novak, Percival D Sampaio-Barros, Cristiane Kayser, Ira Litinsky, Maura Couto.

Contributors Study design: UAW, VKJ. Analysis of data: EGW, VKJ, UAW. Interpretation of data: EGW, VKJ, UAW, OD, PH. Acquisition of data: UAW, YA, GR, $E H, O D, P A, P E C, M T, S V, A B G, N D, U M-L, J D, M L$. All authors were involved in drafting the article or revising it critically for important intellectual content, and all authors approved the final version of the manuscript.

Competing interests None.

Ethics approval Ethics approval has been obtained from all respective local ethics committees.

Provenance and peer review Not commissioned; externally peer reviewed.

Data sharing statement Data are available upon valid request. 


\section{Clinical and epidemiological research}

\section{REFERENCES}

1 Gabrielli A, Avvedimento EV, Krieg T. Scleroderma. N Engl J Med 2009;360:1989-2003.

2 Rodnan GP, Lipinski E, Luksick J. Skin thickness and collagen content in progressive systemic sclerosis and localized scleroderma. Arthritis Rheum 1979;22:130-40.

3 Clements P, Lachenbruch P, Siebold J, et al. Inter and intraobserver variability of total skin thickness score (modified Rodnan TSS) in systemic sclerosis. J Rheumatol 1995;22:1281-5.

4 Kaldas M, Khanna PP, Furst DE, et al. Sensitivity to change of the modified Rodnan skin score in diffuse systemic sclerosis-assessment of individual body sites in two large randomized controlled trials. Rheumatology (Oxford) 2009;48:1143-6.

5 LeRoy EC, Black C, Fleischmajer R, et al. Scleroderma (systemic sclerosis): classification, subsets and pathogenesis. J Rheumatol 1988;15:202-5.

6 Walker UA, Tyndall A, Czirják L, et al. Clinical risk assessment of organ manifestations in systemic sclerosis: a report from the EULAR Scleroderma Trials And Research group database. Ann Rheum Dis 2007;66:754-63.

7 Mouthon L, Mestre-Stanislas C, Bérezné A, et al. Impact of digital ulcers on disability and health-related quality of life in systemic sclerosis. Ann Rheum Dis 2010;69:214-17.

8 Bérezné A, Seror R, Morell-Dubois S, et al. Impact of systemic sclerosis on occupational and professional activity with attention to patients with digital ulcers. Arthritis Care Res (Hoboken) 2011;63:277-85.

9 Sunderkötter C, Herrgott I, Brückner C, et al. Comparison of patients with and without digital ulcers in systemic sclerosis: detection of possible risk factors. Br J Dermatol 2009;160:835-43.

10 Khimdas S, Harding S, Bonner A, et al. Associations with digital ulcers in a large cohort of systemic sclerosis: results from the Canadian Scleroderma Research Group registry. Arthritis Care Res (Hoboken) 2011;63:142-9.

11 Hasegawa M, Imura-Kumada S, Matsushita T, et al. Anti-topoisomerase I antibody levels as serum markers of skin sclerosis in systemic sclerosis. J Dermatol 2013;40:89-93.

12 Alba MA, Velasco C, Simeón CP, et al. Early- versus late-onset systemic sclerosis: differences in clinical presentation and outcome in 1037 patients. Medicine (Baltimore) 2014;93:73-81.

13 Manno RL, Wigley FM, Gelber AC, et al. Late-age onset systemic sclerosis. J Rheumatol 2011;38:1317-25.
14 Hügle T, Schuetz P, Daikeler T, et al. Late-onset systemic sclerosis-a systematic survey of the EULAR scleroderma trials and research group database. Rheumatology (Oxford) 2011;50:161-5.

15 Perera A, Fertig N, Lucas $M$, et al. Clinical subsets, skin thickness progression rate, and serum antibody levels in systemic sclerosis patients with anti-topoisomerase I antibody. Arthritis Rheum 2007;56:2740-6.

16 Meier FMP, Frommer KW, Dinser R, et al. Update on the profile of the EUSTAR cohort: an analysis of the EULAR Scleroderma Trials and Research group database. Ann Rheum Dis 2012;71:1355-60.

17 Masi AT, Rodnan GP, Medsger TA Jr, et al. Preliminary criteria for the classification of systemic sclerosis (scleroderma). Subcommittee for scleroderma criteria of the American Rheumatism Association Diagnostic and Therapeutic Criteria Committee. Arthritis Rheum 1980;23:581-90.

18 Steen VD, Medsger TA. Severe organ involvement in systemic sclerosis with diffuse scleroderma. Arthritis Rheum 2000;43:2437-44.

19 Nikpour M, Hissaria P, Byron J, et al. Prevalence, correlates and clinical usefulness of antibodies to RNA polymerase III in systemic sclerosis: a cross-sectional analysis of data from an Australian cohort. Arthritis Res Ther 2011;13:R211.

20 Kuwana M, Kaburaki J, Mimori T, et al. Autoantibody reactive with three classes of RNA polymerases in sera from patients with systemic sclerosis. J Clin Invest 1993;91:1399-404.

21 Derk CT, Artlett CM, Jimenez SA. Morbidity and mortality of patients diagnosed with systemic sclerosis after the age of 75: a nested case-control study. Clin Rheumatol 2006;25:831-4.

22 Weng $\mathrm{HH}$, Ranganath VK, Oh M, et al. Differences in presentation of younger and older systemic sclerosis patients in clinical trials. Clin Exp Rheumatol 2010;28: S10-14.

23 Hanke K, Dähnrich C, Brückner CS, et al. Diagnostic value of anti-topoisomerase I antibodies in a large monocentric cohort. Arthritis Res Ther 2009;11:R28.

24 Vanthuyne M, Smith V, De Langhe E, et al. The Belgian Systemic Sclerosis Cohort: correlations between disease severity scores, cutaneous subsets, and autoantibody profile. J Rheumatol 2012;39:2127-33.

25 Elhai M, Avouac J, Walker UA, et al. A gender gap in primary and secondary heart dysfunctions in systemic sclerosis: a EUSTAR prospective study. Ann Rheum Dis 2016:75:163-9. 


\section{ARD}

Incidence and predictors of cutaneous manifestations during the early course of systemic sclerosis: a 10-year longitudinal study from the EUSTAR database

Elina G Wirz, Veronika K Jaeger, Yannick Allanore, Gabriela Riemekasten, Eric Hachulla, Oliver Distler, Paolo Airò, Patricia E Carreira, Mohammed Tikly, Serena Vettori, Alexandra Balbir Gurman, Nemanja Damjanov, Ulf Müller-Ladner, Jörg Distler, Mangtao Li, Peter Häusermann and Ulirich A Walker

Ann Rheum Dis2016 75: 1285-1292 originally published online July 31, 2015

doi: 10.1136/annrheumdis-2015-207271

Updated information and services can be found at:

http://ard.bmj.com/content/75/7/1285

These include: Supplementary
Material

References

Email alerting service
Supplementary material can be found at:

http://ard.bmj.com/content/suppl/2016/11/21/annrheumdis-2015-2072 71.DC2

http://ard.bmj.com/content/suppl/2015/07/31/annrheumdis-2015-2072 71.DC1

This article cites 25 articles, 6 of which you can access for free at: http://ard.bmj.com/content/75/7/1285\#ref-list-1

Receive free email alerts when new articles cite this article. Sign up in the box at the top right corner of the online article.

Topic
Collections

Articles on similar topics can be found in the following collections

ARD Lay summaries (83)

Immunology (including allergy) (5144)

Connective tissue disease (4253)

Epidemiology (1413)

\section{Notes}

To request permissions go to:

http://group.bmj.com/group/rights-licensing/permissions

To order reprints go to:

http://journals.bmj.com/cgi/reprintform

To subscribe to BMJ go to:

http://group.bmj.com/subscribe/ 